\title{
On Needles and Haystacks: The Perils of Cardiovascular Risk Screening in Rheumatoid Arthritis
}

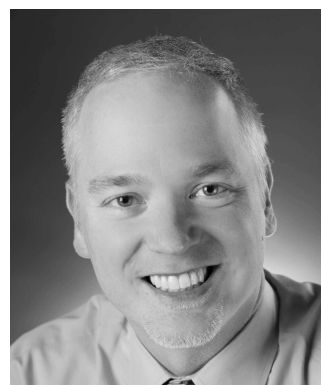

Finding a needle in a haystack is a proverbially difficult task. The chance of finding a needle is increased if the haystack is made up of a substantial proportion of needles, unless the needles are identical in appearance to the hay. This illustrates a challenge in the development of risk prediction models for cardiovascular disease (CVD) events in rheumatoid arthritis (RA). Although there are many needles in the RA population (i.e., individuals with elevated CVD risk appropriate for early preventive intervention), many do not appear to be high risk based on traditional CVD risk factors. As a telling illustration, Crowson, et $a l^{1}$ demonstrated that $16 \%$ of the 525 patients with RA from Olmsted County, Minnesota, USA, experienced a CVD event during 10 years of followup. However, the 10-year Framingham risk score only predicted that $8.7 \%$ would have an event, a striking difference that illustrates the potential contribution of nontraditional risk factors, such as those related to RA disease activity and severity, to CVD risk.

When almost $50 \%$ of the needles look like hay, how do you separate them? An early approach proposed was to multiply an individual's CVD risk score by 1.5 , based on the oft-cited average relative increase in CVD events for RA compared with the general population ${ }^{2,3}$. Although based on expert opinion and not validated using data from a longitudinal cohort, this strategy was recommended by a guideline committee organized by the European League Against Rheumatism Standing Committee for Clinical Affairs. In its first guideline published in $2010^{4}$, the committee recommended using the 1.5 multiplication of the CVD risk score appropriate for the background general population for patients with RA meeting at least 2 of 3 criteria: disease duration exceeding 10 years, seropositivity for rheumatoid factor and/or anticyclic citrullinated protein, or severe extraarticular manifestations. Despite demonstrating little clinical utility in several studies ${ }^{1,5}$, the 1.5 multiplier was again recommended when the guideline was updated in $2015 / 2016^{6}$, this time for all patients with any form of inflam- matory arthritis and with no restriction to those with seropositivity, disease duration, or extraarticular manifestations. No studies have yet been published exploring whether this additional change in the recommendation improves CVD risk prediction.

Multipliers tend to have little effect in those of low/intermediate risk but overestimate those already at higher risk. A more data-driven approach would involve isolating the RA-specific features that are the most predictive and then evaluating their contribution to the model over and above traditional CVD risk factors. With this aim, Solomon, et al leveraged the considerable size of the Consortium of Rheumatology Researchers of North America (Corrona) registry to develop and internally validate a CVD risk prediction model for $\mathrm{RA}^{7}$. The resulting Expanded Risk Score for RA (ERS-RA) was derived from a cohort of more than 23,000 patients with RA who accumulated 347 CVD events over an average of 2.2 years of followup. The final prediction model included traditional CVD risk factors and 4 RA factors: a baseline Clinical Disease Activity Index score $>10$ units (indicating moderate or high disease activity), Health Assessment Questionnaire-Disability Index (HAQ-DI; $>0.5$ units), any prednisone use, and RA disease duration $\geq 10$ years. Within this cohort of RA patients with largely established disease (mean RA duration of about 9 yrs) and a high frequency of treatment with biologic and nonbiologic disease-modifying antirheumatic drugs (DMARD; $>90 \%$ ), the identified RA characteristics improved model discrimination over and above traditional risk factors, with an area under the receiver operator curve (AUC) for predicting CVD events of 0.7609 for the full model. This AUC is on par with the internal validation of the American College of Cardiology/American Heart Association (ACC/AHA) risk calculator for the general population of white and African American men and women in the United States, which ranged from 0.71 to 0.82 depending on sex and race $^{8}$. However, discrimination was lower in an external

See CVD risk prediction in RA, page 130

Personal non-commercial use only. The Journal of Rheumatology Copyright (C) 2019. All rights reserved. 
validation study of ERS-RA that combined 7 multinational longitudinal cohorts made up of 1796 patients with RA who amassed 100 CVD events over an average followup of 6.9 years ${ }^{5}$. ERS-RA did not perform better than the ACC/AHA calculator, which includes no RA-specific features. In addition, the ERS-RA re-categorized more patients with RA from high to low risk who had events $(n=8)$ than it correctly re-categorized from low to high risk those who had an event $(n=6)$. However, there are several factors that may have contributed to the lack of external validation. Although the age of the external validation cohort was similar to that of the Corrona cohort used to develop the ERS-RA, they had a much larger proportion with early RA (almost $60 \%$ with a disease duration $<1 \mathrm{yr}$ ), and any DMARD treatment was much less frequent (36\% with nonbiologic use and only $6 \%$ with biologics). Thus, it is not completely surprising that risk prediction was dissimilar.

A similar potential for incomparability is present for the study by Wahlin, et al reported in this edition of The Journal $^{9}$. They followed 810 patients with RA who all had very early disease at CVD risk determination (i.e., symptom duration of $<1 \mathrm{yr}$ ) who amassed $73 \mathrm{CVD}$ events over an average followup time of 8.5 years. As with the external validation study by Crowson, et $a l^{5}$, the ERS-RA model was not superior to the ACC/AHA model in the prediction of CVD events, even when hypertension and hyperlipidemia were defined based on laboratory variables rather than patient/provider report. However, is it plausible to expect a risk calculator to be equally predictive across an entire span of a patient's disease experience? Traditional CVD risk factors, if left untreated, generally change little over time. However, RA characteristics are time-varying, and may have different implications across the disease span. For example, assessment of physical dysfunction using the HAQ-DI may reflect different processes at different phases of RA. In early RA, the HAQ score is more reflective of articular disease activity (i.e., synovitis) and systemic effects (i.e., fatigue), whereas in later disease there is the potential for greater contribution from articular damage accrual, sarcopenia, and the accompanying effect of both on physical fitness ${ }^{10}$. Glucocorticoid use in early RA is often also a reflection of disease activity and is frequently tapered. However, glucocorticoid use in later disease represents a group likely resistant to tapering who has accumulated extended exposure to the atherogenic effects of hypercortisolism. Thus, it is not at all surprising that the ERA-RA model was not as predictive in early RA because 1 key predictive factor for ERS-RA (RA duration $>10 \mathrm{yrs}$ ) could not be present. In addition, the remaining 3 factors were likely reflective of the same exposure (i.e., synovitis) in the very early group rather than the composite of synovitis, damage, disability, reduced physical fitness, and prolonged exposure to atherogenic glucocorticoids in the established cohort.

Do we need different RA-specific CVD risk calculators for different phases of disease? Do we need RA-specific CVD risk calculators at all, considering that there are no rigorously evaluated primary prevention strategies identified for CVD risk reduction in RA? Although recent reports are encouraging that $\mathrm{CVD}$ event rates are declining in $\mathrm{RA}^{11}$, not all reports reveal normalization to the rates of the background general population, which have also been temporally declining ${ }^{12}$. Although it is unclear whether these trends are related to more aggressive control of inflammatory burden or traditional CVD risk factors, there will likely remain a relevant subgroup of patients who will have RA-driven accelerated atherosclerosis, either from the severity of their disease and/or from delayed treatment or undertreatment. The field has made great strides over the past decade in measuring the magnitude of the burden of CVD in RA and much progress in isolating its determinants. However, without an actionable tool for accurate CVD screening and prevention that can be carried into the RA clinic, our efforts to understand CVD risk in RA will have benefitted few actual patients with RA.

\author{
JON T. GILES, MD, MPH, \\ Associate Professor of Medicine, \\ Division of Rheumatology, \\ Columbia University, \\ College of Physicians and Surgeons, \\ 630 West 168th St., \\ Physicians and Surgeons Building, \\ Suite 3-450, New York, New York 10032, USA.
} Address correspondence to Dr. J.T. Giles

E-mail: jtg2122@columbia.edu

\section{REFERENCES}

1. Crowson CS, Matteson EL, Roger VL, Therneau TM, Gabriel SE. Usefulness of risk scores to estimate the risk of cardiovascular disease in patients with rheumatoid arthritis. Am J Cardiol 2012;110:420-4.

2. Avina-Zubieta JA, Choi HK, Sadatsafavi M, Etminan M, Esdaile JM, Lacaille D. Risk of cardiovascular mortality in patients with rheumatoid arthritis: a meta-analysis of observational studies. Arthritis Rheum 2008;59:1690-7.

3. Avina-Zubieta JA, Thomas J, Sadatsafavi M, Lehman AJ, Lacaille D. Risk of incident cardiovascular events in patients with rheumatoid arthritis: a meta-analysis of observational studies. Ann Rheum Dis 2012;71:1524-9.

4. Peters MJ, Symmons DP, McCarey D, Dijkmans BA, Nicola P, Kvien TK, et al. EULAR evidence-based recommendations for cardiovascular risk management in patients with rheumatoid arthritis and other forms of inflammatory arthritis. Ann Rheum Dis 2010;69:325-31.

5. Crowson CS, Gabriel SE, Semb AG, van Riel P, Karpouzas G, Dessein PH, et al. Rheumatoid arthritis-specific cardiovascular risk scores are not superior to general risk scores: a validation analysis of patients from seven countries. Rheumatology 2017;56:1102-10.

6. Agca R, Heslinga SC, Rollefstad S, Heslinga M, McInnes IB, Peters MJ, et al. EULAR recommendations for cardiovascular disease risk management in patients with rheumatoid arthritis and other forms of inflammatory joint disorders: 2015/2016 update. Ann Rheum Dis 2017;76:17-28.

7. Solomon DH, Greenberg J, Curtis JR, Liu M, Farkouh ME, Tsao P, et al. Derivation and internal validation of an expanded cardiovascular risk prediction score for rheumatoid arthritis: a

Personal non-commercial use only. The Journal of Rheumatology Copyright @ 2019 . All rights reserved. 
Consortium of Rheumatology Researchers of North America Registry Study. Arthritis Rheumatol 2015;67:1995-2003.

8. Goff DC Jr., Lloyd-Jones DM, Bennett G, Coady S, D'Agostino RB, Gibbons R, et al. 2013 ACC/AHA guideline on the assessment of cardiovascular risk: a report of the American College of Cardiology/American Heart Association Task Force on Practice Guidelines. Circulation 2014;129:S49-73.

9. Wahlin B, Innala L, Magnusson S, Möller B, Smedby T, Rantapää-Dahlqvist S, et al. Performance of the Expanded Cardiovascular Risk Prediction Score for Rheumatoid Arthritis is not superior to the ACC/AHA risk calculator. J Rheumatol 2019;46:130-7.

10. Aletaha D, Smolen J, Ward MM. Measuring function in rheumatoid arthritis: Identifying reversible and irreversible components. Arthritis Rheum 2006;54:2784-92.

11. Myasoedova E, Gabriel SE, Matteson EL, Davis JM 3rd, Therneau TM, Crowson CS. Decreased cardiovascular mortality in years: dawn of a new era in cardiovascular disease in RA? J Rheumatol 2017;44:732-9.

12. Holmqvist M, Ljung L, Askling J. Acute coronary syndrome in new-onset rheumatoid arthritis: a population-based nationwide cohort study of time trends in risks and excess risks. Ann Rheum Dis 2017;76:1642-7.

J Rheumatol 2019;46:121-3; doi:10.3899/jrheum.180933 